\title{
DISTRIBUCIÓN POTENCIAL DEL HÁBITAT DEL JAGUAR Y ÁREAS DE CONFLICTO HUMANO-JAGUAR EN LA PENÍNSULA DE YUCATÁN
}

\author{
CUAUHTÉMOC CHÁVEZ Y HELIOT ZARZA \\ Universidad Nacional Autónoma de México, Instituto de Ecología, 3er Circuito exterior s/n \\ Ciudad Universitaria, México, D. F. 04510 \\ correoelectronico: cchavez@ecologia.unam.mx
}

\begin{abstract}
Resumen: Las Selvas Mayas de la Península de Yucatán, México, mantienen una de las poblaciones más importantes de jaguar (Panthera onca) al norte de su distribución. Sin embargo, la región está sujeta a grandes cambios en su cobertura forestal debido al desarrollo humano y agropecuario de los últimos años. Esto ha ocasionado una reducción en el hábitat del jaguar y por consiguiente un incremento en la incidencia de ataques al ganado doméstico por jaguares, debido a ello es necesario contar con información sólida sobre las áreas de conflicto humano-jaguar para ser consideradas durante los planes de manejo y conservación a nivel regional. Nuestro análisis se basó en un grupo de variables (vegetación, uso de suelo, precipitación y clima) y una base de datos georeferenciados tanto de presencia de jaguares como de depredación de ganado domestico en la Península de Yucatán. Nosotros demostramos la posibilidad de modelar la distribución potencial de los sitios de conflicto humano-jaguar usando el método de máxima entropía (MaxEnt). Entre las variables usadas la vegetación demostró ser la variable que mejor explica la distribución potencial del jaguar. En cambio, el modelo de conflictos humano-jaguar muestra que el mayor número de casos de depredación se presenta alrededor de los asentamientos humanos, en ambientes dominados por actividades humanas. Modelar la distribución potencial de las áreas de conflicto humano-jaguar ofrece una gran oportunidad a los biólogos de la conservación y tomadores de decisiones para poder diseñar estrategias regionales para mitigar el conflicto con los depredadores con base en la distribución espacial potencial de los ataques.
\end{abstract}

Palabras clave: Hábitat potencial, jaguar, depredación, Yucatán.

Abstract: The Mayan Forest of the Yucatan Peninsula, Mexico, maintain an of the most important populations of jaguar (Panthera onca) north of his distribution. However, the region is subject to big changes in this forest coverage because of the human and agriculture-livestock development of the last years. This has caused a reduction in the habitat of the jaguar and therefore an increase in the incidence of attacks to the domestic livestock by jaguars, because of this is necessary to have solid information on the areas of human-jaguar conflict to be considered during the management plans and conservation to regional level. Our analysis based in a group of variable (vegetation, use of soil, precipitation and climate) and a base of georeference data so much of presence of jaguars and of livestock depredation in the Yucatan Peninsula. We showed the possibility of model the potential distribution of the places of human-jaguar conflict using the method of maximum entropy (MaxEnt). Between the variables 
used the vegetation showed to be the variable than better explains the potential distribution of the jaguar. Instead, the model of human conflicts-jaguar show that the main number of cases of depredation present around the human settlements, in landscape dominated by human activities. Modeling the potential distribution of the areas of human-jaguar conflict- offers a big opportunity to the biologists of the conservation and decisions-makers to can design regional strategies to mitigate the conflict with the predatory with base in the spatial distribution potential of the attacks.

Key words: Potencial habitat, jaguar, predation, Yucatán.

\section{INTRODUCCION}

Las poblaciones de grandes carnívoros han declinado globalmente desde el siglo pasado debido a la expansión de las actividades humanas y/o a los conflictos con el hombre en todas las regiones donde ambos coexisten (Woodroffe, 2000). Entre los conflictos humano-carnívoro, la depredación del ganado doméstico se ha convertido en un serio problema que amenaza la conservación de los grandes felinos (Treves y Karanth, 2003), como es el caso del león (Panthera leo), tigre (Panthera tigris), pantera de las nieves (Uncía uncia), puma (Puma concolor) y jaguar (Panthera onca; Kurten y Anderson, 1980; Ceballos et al., 2007). En la mayoría de los casos, la solución inmediata al problema es eliminar al depredador, ante la falta de otras posibles soluciones (Patterson et al., 2004).

México mantiene numerosas poblaciones de jaguar en el sureste del país, siendo la más importante la localizada en las Selvas Mayas de la Península de Yucatán, región considerada de alta prioridad de conservación para la especie (Sanderson et al., 2002; Chávez y Ceballos, 2006) y un "hotspot" por su alta biodiversidad (Myers et al., 2000). Sin embargo, esta región, históricamente ha estado sometida a una intensa actividad humana y transformación de su paisaje a lo largo de su historia y especialmente en el último siglo, provocando grandes cambios en el uso del suelo de la región (Boege, 1995; Roy Chowdhury, 2006; Vester et al.,2007).

El número creciente de quejas sobre la depredación de ganado y la alta mortalidad de jaguares, requiere de soluciones inmediatas. Para ello se necesita identificar los sitios que potencialmente son más susceptibles a sufrir ataques de depredación de ganado por parte de los jaguares y con ello dirigir esfuerzos y recursos para mitigar la depredación de ganado en esta región. Para cumplir con este objetivo, fue necesario determinar la distribución potencial del jaguar e identificar las áreas con mayor potencialidad a presentar conflicto humano-jaguar y finalmente evaluar el papel de las áreas protegidas como estrategia de conservación del jaguar en la región en la Península de Yucatán. 


\section{AREADEESTUDIO}

El estudio se realizó en la Península de Yucatán, que comprende los estados de Campeche, Quintana Roo y Yucatán. Se delimitó con las siguientes coordenadas geográficas $21^{\circ} 12^{\prime}$ a $17^{\circ} 49^{\prime} \mathrm{N}$ y $91^{\circ} 00^{\prime}$ a $86^{\circ} 40^{\prime} \mathrm{O}$; cubre una superficie aproximada

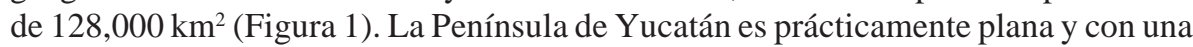
topografía uniforme (0 a 400 m.s.n.m); la principal serranía es el Sierrita de Ticul al sur de Yucatán. Los suelos dominantes de la región son las rendizcas, vertisoles y litosoles. El clima es tropical subhúmedo, la temperatura media anual es de $24.6^{\circ} \mathrm{C}$, con una marcada estacionalidad concentrándose las lluvias en el verano (junio-noviembre). La Península de Yucatán se caracteriza por la ausencia casi total de ríos o arroyos permanentes. El tipo de vegetación dominante es la selva alta y mediana subperennifolia, seguida de la selva mediana caducifolia. En la línea costera, el manglar y las sabanas son la vegetación predominante (SEMARNAP, 2000).

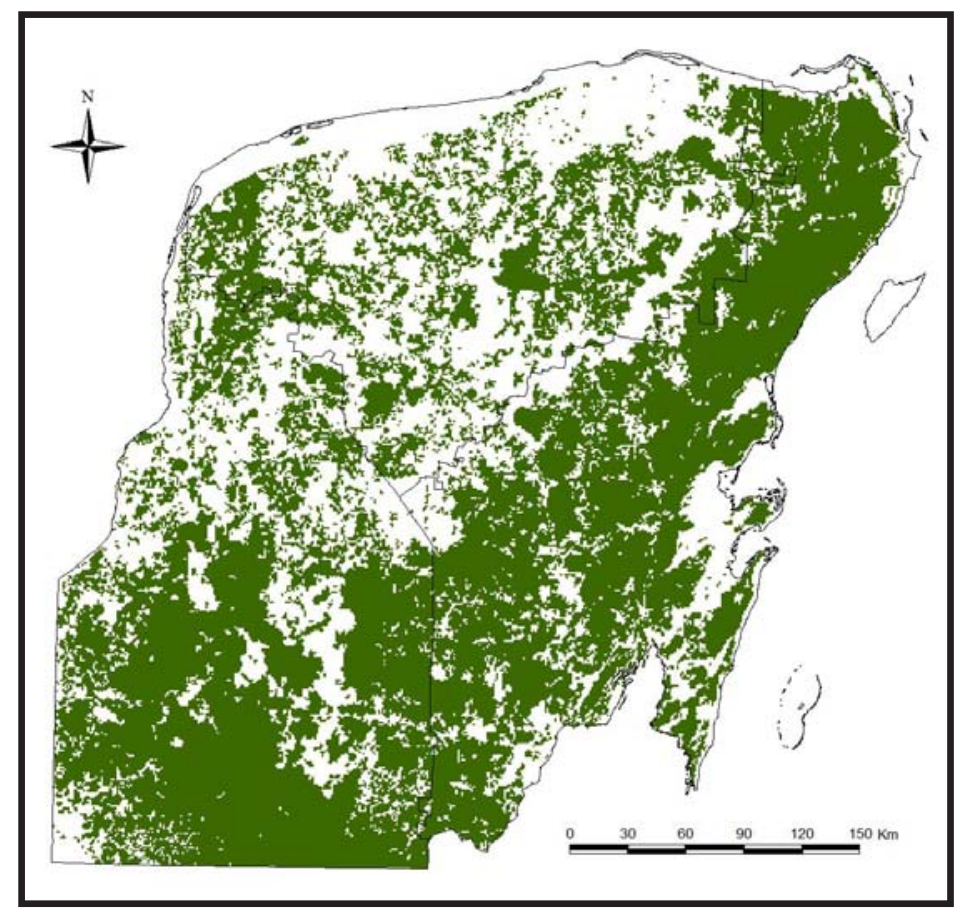

Figura 1. Hábitat potencial del jaguar en la Península de Yucatán generado por Maxent. 
Los ambientes modificados son todos aquellos que han perdido parcialmente su cobertura vegetal natural y/o que presentan vegetación secundaria. Estos tipos de hábitats se encuentran asociados a los campos agrícolas, pastizales inducidos y por lo general se localizan alrededor de los asentamientos humanos. Los asentamientos humanos y las áreas agropecuarias ocupan aproximadamente el 24\% de la superficie de la Península. La actividad pecuaria asciende a 898,393 cabezas de ganado bovino, de las cuales más del 60\% de las existencias están dentro del sistema de producción de libre pastoreo (INEGI, 2009).

\section{METODOS}

\section{Composición del paisaje}

Se determino la identidad de los principales tipos de vegetación, superficie y número de fragmentos por cada tipos de vegetación, así como el grado de fragmentación de cada uno de ellos, a partir del Inventario Nacional Forestal 2000-2001 (SEMARNAP et al., 2000), utilizando el programa Arcview 3.2 (ESRI, 1996; Cuadro 1).

Cuadro 1. Tipos de vegetación y uso de suelo presente en la Península de Yucatán.

\begin{tabular}{lrc}
\hline $\begin{array}{l}\text { Tipos de vegetación } \\
\text { y uso de suelo }\end{array}$ & Superficie (ha) & Número de Parches \\
\hline Selva Alta y Mediana & $3,870,554$ & 272 \\
Selva Alta y Mediana & 884,018 & 511 \\
con vegetación secundaria & & 155 \\
Selva Mediana Caducifolia & $1,116,698$ & 378 \\
Selva Mediana Caducifolia & $1,184,302$ & \\
con vegetación secundaria & & 506 \\
Selva Baja Subperennifolia & 726,242 & 247 \\
Selva Baja Subperennifolia & 211,131 & 45 \\
con vegetación secundaria & & 108 \\
Selva Baja Caducifolia & 198,293 & \\
Selva Baja Caducifolia & 645,960 & 233 \\
con vegetación secundaria & & 193 \\
Popal-tular & 321,570 & 107 \\
Manglar & 311,003 & 1,479 \\
Otros tipos de vegetación & 74,689 & 377 \\
Pastizal & $1,674,941$ & 61 \\
Área urbana & 77,101 & \\
Área sin vegetación aparente & 36,058 & \\
\hline
\end{tabular}




\section{Datos de ocurrencia}

Se generó una base de datos con todos los registros de presencia verificada de jaguar a partir de 1990 en la región $(n=25)$ y otra con los registros verificados de ataque de jaguar a ganado domésticos $(n=45)$ entre 1998 al 2004, estos datos provienen de distintas fuentes (Apéndice). En el caso de la base de datos de ataques se usaron solamente 23 registros ya que se descartaron aquellas localidades imprecisas (e.g., Hunucmá).

\section{Variables ambientales}

Se emplearon tres coberturas ambientales para la construcción del modelo de distribución potencial del jaguar y de las posibles áreas de conflicto. La cobertura de vegetación y uso de suelo proviene del Inventario Nacional Forestal 2000-2001, a una escala de 1:250,000 (SEMARNAP et al., 2000). Las coberturas de precipitación y edafología provienen de la Comisión Nacional para el Conocimiento y Uso de la Biodiversidad (CONABIO), escala de 1:1,000,000. Todos los datos y coberturas se transformaron a formato raster con una resolución de 1 km².

\section{Modelación}

Se usó el método de Máxima Entropía - MaxEnt versión 2.3 (Phillips et al, 2006, software libre disponible en www.cs.princeton.edu/ schapire/maxent), porque tiene un mejor comportamiento en la modelación de la distribución del hábitat potencial (Ortega-Huerta y Peterson 2008) y es robusto a pesar de tamaños de muestra pequeños (Benito et al., 2009). Maxent es un programa que estima la distribución de probabilidad de ocurrencia de las especies a partir de las limitantes medioambientales (Phillips et al., 2006).

Para correr los modelos distribución potencial del jaguar y las áreas de conflicto humano-jaguar en la Península de Yucatán, se emplearon los valores preestablecidos del programa con un umbral ("threshold") de $10^{-5}$ y 500 iteraciones. El valor de regularización empleado fué de 0.25 y se utilizó la opción del tipo de distribución Lineal Cuadrática (Feature types: Linear Quadratic) dado que el tamaño de muestra fue pequeño (Phillips et al., 2006).

Para la validación y adecuación del modelo se utilizó el área bajo la curva (AUC), donde se ve el desempeño y ajuste del modelo para predecir las presencias contenidas en la muestra de los datos. Para determinar las variables más importantes en el modelo, se corrió una prueba de jackknife para medir la importancia de cada una de ellas. 


\section{RESULTADOS Y DISCUSIÓN}

\section{Composición del paisaje}

La vegetación natural conservada se distribuye en gran parte de la Península de Yucatán (46\%), y aún se conservan numerosos fragmentos mayores a 100 ha $(<10$ ha $=$ 646 ; $>10<100$ ha $=280$; $>100$ ha $=52$ ). La vegetación natural con vegetación secundaria ocupa el 23\% de la superficie de la Península (1,244 parches). En comparación, los ambientes modificados representan el 25\% del área total y a diferencia de la vegetación natural presenta una mayor fragmentación $(2,898$ parches, $<10$ ha $=928 ;>10<100$ ha $=$ $188 ;>100$ ha $=17$, Cuadro 1). El uso de suelo dominante en los ambientes modificados es el pecuario (13\%) y agrícola (11\%).

Los resultados indican que la región todavía mantiene una gran superficie de vegetación natural, en comparación con las estimaciones para Mesoamérica, donde cerca del $80 \%$ de las cobertura forestal se ha perdido o modificado a una tasa de deforestación de 1.4 a 2.5\% anual (CEPF, 2001).

\section{Distribución potencial del hábitat del jaguar}

El modelo predice una gran extensión de hábitat potencial para el jaguar (> 54 mil km²) para la Península de Yucatán, compuesta por selvas altas y medianas, con una precipitación anual promedio de 1,200-1,500 mm y predominantemente en suelo del tipo cambisol y vertisol.

El modelo obtenido indica que la distribución potencial del jaguar en la Península de Yucatán no es aleatoria (área bajo la curva AUC=0.94, Intervalo de confianza 95\%; porcentaje de presencias correctamente clasificadas \%PCC = 86\%). El análisis de Jackknife sugiere que la variable que contribuye más al modelo es la vegetación.

El 42\% de la superficie de la Península de Yucatán son áreas con una alta probabilidad de condiciones adecuadas para la presencia de jaguar. A nivel estatal, Quintana Roo presenta la mayor superficie (57\%) y en promedio los parches de mayor tamaño (Cuadro 2). Sin embargo, estas áreas enfrentan diversos escenarios. Por ejemplo, la parte norte de Quintana Roo, afronta serios problemas de conectividad con la parte sur principalmente por la creación, ampliación de infraestructura para el desarrollo turístico (Faller et al., 2007; Navarro et al., 2007). Mientras que la parte sur, enfrenta un avance de la frontera pecuaria y agrícola (Chávez et al., 2007; Zarza et al., 2007).

Más del 60\% de la distribución potencial del jaguar predicha está conformada por parches menores a $10 \mathrm{~km}^{2}$ (Cuadro 2). Existen algunos parches $>100$ ha que 
facilitan la conectividad, en los cuales deben promoverse estrategias de conservación, como el pago de servicios ambientales (i.e. captura de carbono, mantenimiento de la Biodiversidad) o el desarrollo de proyectos productivos compatibles con la conservación de los recursos naturales (i.e. áreas forestales certificadas, apicultura, artesanías), mitigando el impacto del cambio en el uso del suelo en la región (Zarza, 2008). La viabilidad de la población de jaguar en la región, dependerá de su adaptación a los patrones y procesos de fragmentación de su hábitat (Morrison et al., 1992). Sin embargo, estos aún no han podido ser determinados con detalle, por lo que es una prioridad conocer hasta que punto los jaguares pueden sobrevivir en un paisaje dominado por actividades humanas.

\section{Distribución potencial del Conflicto Humano-Jaguar}

Las áreas identificadas con mayor probabilidad de conflicto humano-jaguar en la Península de Yucatán son aquellas cercanas a los asentamientos humanos y cuerpos de agua, caracterizadas por una precipitación promedio anual de 1,000-1,200 mm y suelos cambisoles y luvisoles (Figura 2).

El modelo identificó áreas importantes de conflicto ganadero en la Península $($ AUC $=0.94 ; \mathrm{IC}=0.95 \%$; \%PC $=88 \%)$. En el estado de Quintana Roo, se identificaron dos áreas importantes, una al norte cerca de Cancún y la otra al noroeste de Felipe Carrillo Puerto; para Yucatán, se localiza en la zona de influencia de la Ciudad de Mérida, una segunda área al sureste de Tekax y Peto, y una tercera en la región sur de Ría Largartos; finalmente Campeche, presenta varios puntos de conflicto alrededor de la Reserva de la Biosfera de Calakmul, y de las áreas protegidas Balam kin y Balam ku, otro punto potencial de conflicto es la parte centro del estado, a la altura de Pustunich, Etzna y Pizayal.

Las áreas identificadas como de conflicto humano-jaguar ocupan el 14 \% de la superficie total de la Península de Yucatán. El estado con la mayor área potencial de ataques de jaguar es Yucatán, seguido de Campeche (Cuadro 2). Históricamente, la principal actividad económica del estado de Yucatán era la agrícola, actualmente es uno de los estados con mayor producción porcina y bovina del país (Sánchez y Rebollar, 1999). A pesar del gran cambio ambiental en está región, aún se mantienen grandes extensiones forestales de propiedad ejidal que permanecen en buen estado de conservación (SEMARNAP, 2000).

A pesar de lo anterior, el cambio en la cobertura forestal, provocada por una mayor demanda en espacio y recursos naturales por la población humana, aumenta las posibilidades de contacto con la fauna silvestre y con ello el conflicto con los carnívoros (i.e. jaguar y puma). Debido a ello existe la necesidad de tener un protocolo estandarizado para evaluar el conflicto ganadero-carnívoros para las condiciones existentes en México (Chávez y Ceballos, 2006). 


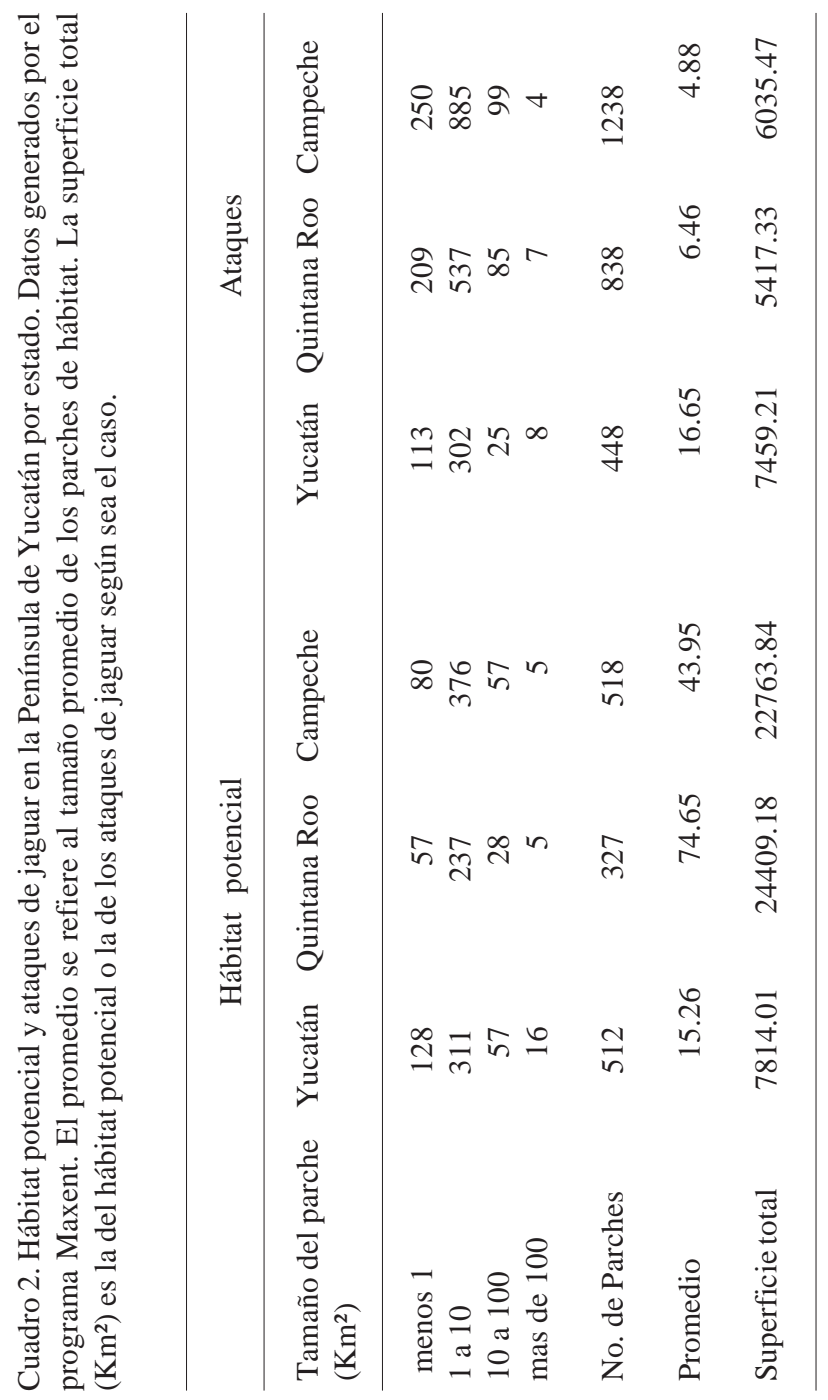




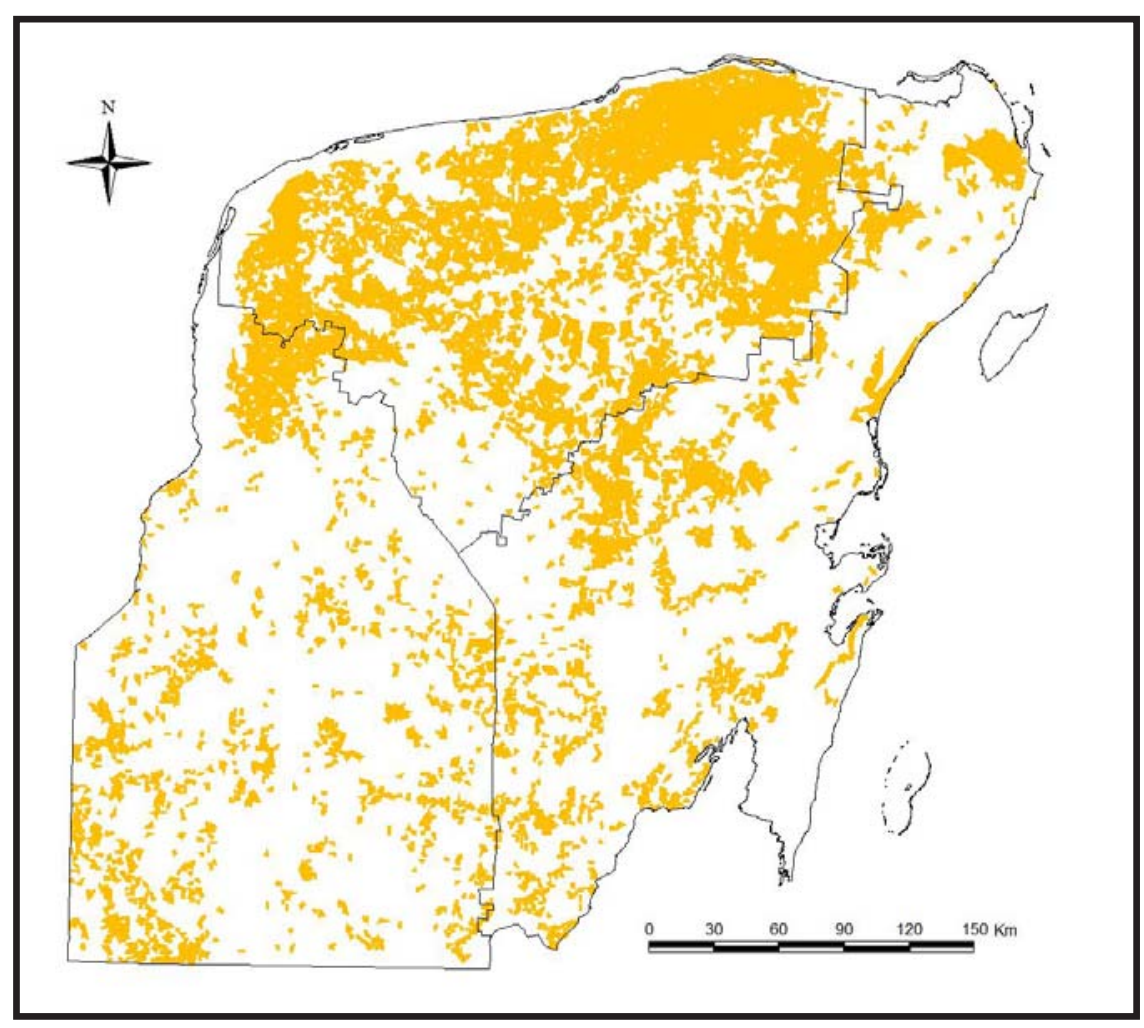

Figura 2. Ataques potenciales del jaguar en la Península de Yucatán generado por Maxent.

Existe un protocolo de acción para evaluar la depredación del ganado doméstico (Hoogeisteijn, 2003; www.wcs.org), el cual no refleja las condiciones existentes en muchos lugares de México. Se implementó un programa a nivel estatal por Gobierno del Estado de Yucatán, cual fue dirigido específicamente a los rancheros, para compensarlos económicamente si sufrían perdidas en su ganado doméstico a consecuencia de la depredación por jaguar el cual no tuvo éxito, debido a la falta de asesoría, recursos económicos y humanos (C. Alcerreca y A. González-Terrazas, com. pers.). En Quintana Roo, se realizó un programa piloto de compensación por depredación de ganado en el Ejido Caoba, Municipio de Othón P. Blanco, implementado por Unidos para la Conservación, Sierra Madre, Instituto de Ecología de la Universidad Nacional Autónoma de México y Ecosafari, donde se elaboró un 
protocolo para determinar el origen del ataque, además del personal calificado para la evaluación de los daños (Chávez et al., obs. no publicados).

\section{Traslape de la distribución espacial del jaguar y conflicto humano-jaguar}

Al traslapar el mapa de hábitat potencial de jaguar y de conflicto humano-jaguar se observa que una parte del hábitat potencial se encuentra en las áreas naturales protegidas, y algunas de ellas están en vías de aislamiento por las actividades humanas, lo que potencia el riesgo de conflicto humano-jaguar en la zona de amortiguamiento y borde de dichas áreas protegidas. Esto es lo que sucede con la Reserva de la Biosfera Calakmul la cual mantiene el mayor hábitat disponible para jaguar (5,146 km²); sin embargo en su periferia y área de amortiguamiento se identifican sitios potenciales de conflicto humano-jaguar (893 km², Cuadro 3). Por otra parte, la mayor parte (casi el 80\%) del hábitat potencial del jaguar se encuentra fuera de las ANP's, se ubica en tierras ejidales, de ahí resalta la importancia de los ejidos forestales del centro y oeste del sur de la Península de Yucatán, que permiten la conexión entre las grandes reservas de la región (Reserva de la Biosfera de Calakmul y Sian ka’an, Figura 3). Estas reservas y los grandes fragmentos representan en total un área de hábitat base para la conservación del jaguar en la Península de Yucatán (Ceballos et al., 2002; Chávez, 2006; Chávez et al., 2007; Zarza et al., 2007).

Los esfuerzos de conservación deberán ser dirigidos aquellas áreas que puedan complementar el papel de las ANP's existentes, sin excluir a los dueños de la tierra. Será muy importante la generación de mecanismos e incentivos económicos para la conservación de los recursos naturales en general, y en particular para el hábitat del jaguar. Sin embargo, la incertidumbre en la toma de decisiones entre los diferentes niveles de gobierno, pueden hacer que los planes de conservación no se concreten. Adicionalmente, el desarrollo turístico de algunos lugares lleva a la creación de infraestructura y asentamientos humanos, que si no existe una buena planeación disminuirá de manera importante el hábitat del jaguar (Conde et al., 2007). Sin embargo, el gran reto que enfrenta la conservación a este nivel es que se incentiven aquellas áreas fuera de las ANP's, que mantienen una buena proporción de hábitat del jaguar, así como el mantenimiento de procesos ecológicos y de servicios ambientales para un beneficio común de la población humana.

Este estudio muestra que existe la necesidad de desarrollar un plan regional para la conservación de la fauna silvestre en las áreas de hábitat para jaguar identificadas en la Península de Yucatán, involucrando la colaboración de los diversos actores como poseedores de tierra (grupos individuales), inversionistas y desarrolladores, académicos e instancias del gobiernos, lo cual refuerza las estrategias propuestas en el Plan Ecoregional de las Selvas Maya, Zoque y Olmeca (Secaira y Maldonado, 2006). 


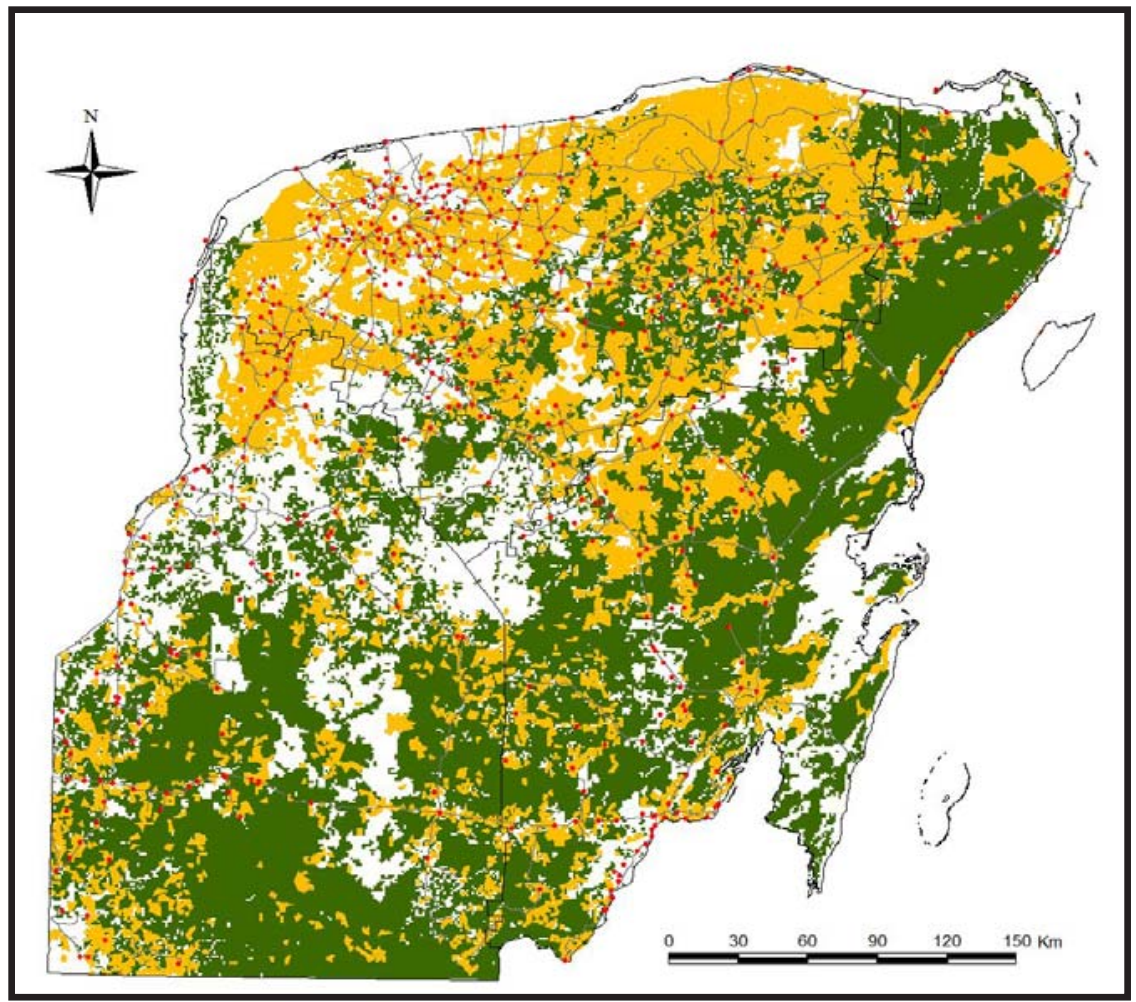

Figura 3. Combinación del hábitat y ataques potenciales del jaguar generado por Maxent en la Península de Yucatán. Los puntos indican los poblados mayores de 200 habitantes.

Además de evaluar, las condiciones del paisaje para el jaguar hacia una comprensión mejor de patrones regionales de la biodiversidad y de planes de colaboración para la conservación entre terratenientes (e.g., ganaderos) en la Península de Yucatán. Algunas instituciones y personas están elaborando el protocolo para actuar en casos de depredación de ganado en México. De hecho, recientemente se aprobó un proyecto para tratar de generar un diagnóstico en seis áreas prioritarias para evaluar el conflicto ganadero (O. Ramírez y D. Azuara, com. pers.). 
Cuadro 3. Hábitat potencial y ataques de jaguar en las áreas naturales protegidas en Península de Yucatán. Datos generados por el programa Maxent. Todas las áreas están en Km². Entre paréntesis esta puesto el porcentaje que cubre cada uno de la superficie total de la Reserva.

\begin{tabular}{|c|c|c|c|}
\hline Nombre de la Reserva & Superficie & Hábitat potencial & Ataques \\
\hline YUM BALAM & 1539.54 & $\begin{array}{l}81.27 \\
(5.28)\end{array}$ & $\begin{array}{c}2.34 \\
(0.15)\end{array}$ \\
\hline RIA LAGARTOS & 603.73 & $\begin{array}{c}9.87 \\
(1.63)\end{array}$ & $\begin{array}{l}128.74 \\
(21.32)\end{array}$ \\
\hline PUNTA NIZUC & 25.54 & 0.00 & $\begin{array}{c}0.21 \\
(0.82)\end{array}$ \\
\hline $\begin{array}{l}\text { A. DE PUERTO } \\
\text { MORELOS }\end{array}$ & 3340.17 & $\begin{array}{c}0.01 \\
(0.00)\end{array}$ & $\begin{array}{l}0.21 \\
(0.01)\end{array}$ \\
\hline RIA CELESTUN & 597.06 & 0.00 & $\begin{array}{c}2.00 \\
(0.33)\end{array}$ \\
\hline LOS PETENES & 2832.05 & $\begin{array}{c}0.23 \\
(0.01)\end{array}$ & $\begin{array}{r}2.78 \\
(0.10)\end{array}$ \\
\hline SIAN KAAN & 5279.76 & $\begin{array}{c}1385.04 \\
(26.23)\end{array}$ & $\begin{array}{l}301.82 \\
(5.72)\end{array}$ \\
\hline UAYMIL & 890.75 & $\begin{array}{l}570.62 \\
(64.06)\end{array}$ & $\begin{array}{l}90.58 \\
(10.17)\end{array}$ \\
\hline CALAKMUL & 7227.33 & $\begin{array}{r}5146.29 \\
(71.21)\end{array}$ & $\begin{array}{l}893.82 \\
(12.37)\end{array}$ \\
\hline BALAM KU & 4085.48 & $\begin{array}{r}3143.13 \\
(76.93)\end{array}$ & $\begin{array}{l}442.07 \\
(10.82)\end{array}$ \\
\hline BALAM KIN & 996.06 & $\begin{array}{l}376.55 \\
(37.80)\end{array}$ & $\begin{array}{c}58.07 \\
(5.83)\end{array}$ \\
\hline BALAM KA'AX & 1288.12 & $\begin{array}{l}632.43 \\
(49.10)\end{array}$ & $\begin{array}{l}78.57 \\
(6.10)\end{array}$ \\
\hline Total & 28705.58 & $\begin{array}{r}11345.43 \\
(6.97)\end{array}$ & $\begin{array}{r}2001.21 \\
(39.52)\end{array}$ \\
\hline
\end{tabular}

Es importante notar que dos terceras partes de estas áreas protegen menos de 10.000 hectáreas; únicamente 38 áreas protegen más de 50.000 hectáreas.

\section{CONCLUSIONES}

Modelar la distribución del hábitat del jaguar puede generar mapas de detalle fino que contribuyan en la toma de decisiones de conservación y manejo de la biodiversidad, 
así como dirigir incentivos en las áreas identificadas de conflicto humano-jaguar. Los mapas de distribución de especies pueden ayudar a identificar nuevas áreas que por limitaciones en campo son poco estudiadas y permite implementar alguna estrategia local de conservación. Sin embargo, siempre será necesario la validación en campo a escala local, para no subestimar la información.

Encontrar alternativas de manejo que puedan reducir el conflicto humanojaguar es imperativo para la conservación del jaguar y especialmente en los ambientes modificados. Una medida relativamente simple como puede ser el manejo ganadero puede reducir sustancialmente la depredación del ganado (Crawshaw, 2004;Sáenz y Carrillo, 2002; Zimmermann et al., 2005). Por ejemplo, evitar el libre pastoreo en el interior de los fragmentos de selva (Azevedo y Murray, 2007).

"las soluciones que no involucren los intereses de la comunidad serán inútiles, ya que sólo la gente local tiene la posibilidad de mejorar la citación ambiental a través de cambios en su comportamiento o el de los carnívoros"

Claudio Sillero

\section{AGRADECIMIENTOS}

A las comunidades locales que se encuentran en la Península de Yucatán. En especial al Dr. Ceballos por su apoyo en las distintas fases de este proyecto. Al Instituto de Ecología de la UNAM, a la CONANP. A la Fundación Banco Bilbao Vizcaya por su apoyo financiero. Antonio Rivera, Francisco Zavala, Melissa López, Marcela Araiza por su ayuda en el trabajo de campo. A las siguientes organizaciones Biocenosis A. C., Reserva Ecológica El Edén A. C., Pronatura Península de Yucatán por facilitarnos información, a Luis Pereyra, Carlos Alcerreca, Alejandro González-Terrazas, Marco Lazcano, y Juan Carlos Faller. A los dos revisores del artículo que con sus comentarios lo enriquecieron. Y en especial el apoyo de Osiris Gaona y Jesús Pacheco.

\section{LITERATURACITADA}

Azevedo, F.C.C., y Murray, D.L. 2007. Evaluation of potential factors predisposing livestock to predation by jaguars. Journal of Wildlife Management, 71:2379-2386.

Benito, B.M., Martínez-Ortega, M.M, Muñoz, L.M., Lorite, J., y J. Peñas. 2009. Assessing extinction-risk of endangered plants using species distribution models: a case study of habitat depletion caused by the spread of greenhouses. Biodiversity and Conservation, 18:2509-2520

Boege, E. 1995. The Calakmul Biosphere Reserve (Mexico). Working Paper No. 13. UNESCO (South-South Cooperation. Programme). Paris, Francia.

Ceballos G., Chávez, C., Rivera, A., Manterola, C. y W. Wall. 2002. Tamaño poblacional y conservación del jaguar en la Reserva de la Biosfera de Calakmul, Campeche, México. Pp 
403-418, en: El jaguar en el nuevo milenio: una evaluación de su condición actual, historia natural y prioridades para su conservación. (Medellín, R.A., C. Equihua, C. Chetkiewics, A Rabinowitz, P. Crawshaw, K. Redford, J.G. Robinson, E. Sanderson y A. Taber, eds). Fondo de Cultura Económica, Universidad Nacional Autónoma de México y Wildlife Conservation Society, México D.F.

Ceballos, G., Chávez, C., List, R. y H. Zarza. 2007. Conservación y manejo del jaguar en México: estudios de caso y perspectivas. Conabio - Alianza WWF- Telcel - Universidad Nacional Autónoma de México, México D.F.

Comisión Nacional para el Conocimiento y Uso de la Biodiversidad (CONABIO). 1998. "Curvas de nivel para la República Mexicana". Escala 1:250 000. Extraído del Modelo Digital del Terreno. Instituto Nacional de Estadística, Geografía e Informática (INEG). México D.F.

CEPF. 2001. Ecosistema forestal de Vilcabamba-Amboró del área prioritaria de conservación de la biodiversidad en los Andes tropicales Perú y Bolivia. 38 pp.

http:/www.cepf.net/ImageCache/cepf/content/pdfs/final_2espanish_2emesoamerica_2esouthemmesoamerica_2eep_2epdf/ v1/final.spanish.mesoamerica.southernmesoamerica.ep.pdf

Chávez, C. 2006. Ecología poblacional y conservación del jaguar (Panthera onca) en la Reserva de la Biósfera de Calakmul. Campeche. Maestría en Ecología y Ciencias Ambientales, Facultad de Ciencias, Universidad Nacional Autónoma de México.

Chávez, C. y G. Ceballos (eds). 2006. El jaguar mexicano en el Siglo XXI: situación actual y manejo. CONABIO - UNAM - Alianza WWF Telcel. México D. F.

Chávez, C., Amín, M. y G. Ceballos. 2007. Ecología poblacional del jaguar y sus implicaciones para la conservación en la Península de Yucatán. Pp. 91 -100, en: Conservación y manejo del jaguar en México: estudios de caso y perspectivas ( G. Ceballos, C. Chávez, R. List y H. Zarza, eds). Conabio - Alianza WWF- Telcel - Universidad Nacional Autónoma de México, México D.F.

Chávez, C., Araiza, M., Rivera, A., Manterola , C. y G. Ceballos. 2002. Protocolo de captura y manejo de grandes felinos del "Programa para la Conservación y Manejo del jaguar y puma en la Reserva de la Biosfera de Calakmul, Campeche y ejidos forestales del estado de Quintana Roo". Manuscrito inédito.

García, E. - Comisión Nacional para el Conocimiento y Uso de la Biodiversidad (CONABIO). 1998. "Climas" (clasificación de Koppen, modificado por García). Escala 1:1 000000. México.

Garcia, E. - CONABIO, 1998. "Precipitación total anual". Escala 1: 1000 000. México.

Conde, D., I. Burgues, L.C. Fleck, C. Monterola, yJ. Reid. 2007. Análisis ambiental y económico de proyectos carreteros en la Selva Maya, un estudio regional. Serie Técnica \#10, Conservation Strategy Fund, Arcata, California.

Crawshaw, J. 2004. Depredation of domestic animals by large cats in Brazil. Human Dimensions of Wildlife, 9:329-330.

Environmental Systems Research Institute. 1996. ArcView GIS. Redlands, CA.

Faller, J.C., Chávez, C. Johnson, S.y G. Ceballos. 2007. Densidad y tamaño de la población de jaguar en el noreste de la Península de Yucatán. Pp. 111-122, en: Conservación y manejo del jaguar en México: estudios de caso y perspectivas (G. Ceballos, C. Chávez, R. List y H. 
Zarza, eds). Conabio - Alianza WWF- Telcel - Universidad Nacional Autónoma de México, México D.F.

Instituto Nacional de Estadística y Geografía (INEGI). 2009. Censo Agropecuario 2007. VIII Censo Agrícola, Ganadero y Forestal. Aguascalientes, México.

Instituto Nacional de investigaciones Forestales y Agropecuarias (INIFAP) - Comisión Nacional para el Conocimiento y Uso de la Biodiversidad (CONABIO). 1995. "Edafología". Escalas 1:250 000 y 1:1 000 000. México D.F.

Kurtén B y E. Anderson 1980. Jaguar, Panthera onca. Studer's Cheetah, Acionyx studeri. American Cheetah, Acinonyx trumani. Lake Cat, Felis lacustris. Ocelot, Felis pardalis. River Cat, Felis amnicola. Jaguarundi, Felis yagouaroundi. Pp. 192-195, en: Pleistocene Mammals of North America. New York: Columbia University Press.

Martínez. M., y C. Galindo-Leal. 2002. La vegetación de Calakmul, Campeche, México: Clasificación, descripción y distribución. Boletín de la Sociedad Botánica, 71:7-32.

Morrison, M.L., B.G., Marcot y R.W, Mannan. 1992. Wildlife-habitat relationships: concepts and applications. The University of Wisconsin Press, Madison.

Myers, N., R. A. Mittermeier, G. d.F. y J. Kent. 2000. Biodiversity hotspots for conservation priorities. Nature, 403:853-854.

Navarro, C.J., Remolina J.F. y J.J. Pérez. 2007. El jaguar en Yum Balam y el Norte de Quintana Roo. Pp. 123-132, en: Conservación y manejo del jaguar en México: estudios de caso y perspectivas (G. Ceballos, C. Chávez, R. List y H. Zarza, eds.). Conabio - Alianza WWFTelcel - Universidad Nacional Autónoma de México, México D.F.

Ortega-Huerta, M.A. y A.T. Peterson. 2008. Modeling ecological niches and predicting geographic distributions: A test of six presence-only methods. Revista Mexicana de la Biodiversidad, 79:205-216.

Patterson, B.D. , S.M. Kasiki, E. Selempo y R.W. Kays. 2004. Livestock depredation by lions (Panthera leo) and other carnivores on ranches neighboring Tsavo National Parks, Kenya. Biological Conservation, 119:507-516.

Phillips, S. J., R.P. Anderson, y R.E. Schapire. 2006. Maximum entropy modeling of species geographic distributions. Ecological Modelling, 190:231-259.

Roy Chowdhury, R. 2006. Landscape change in the Calakmul Biosphere Reserve, Mexico: Modeling the driving forces of smallholder deforestation in land parcels. Applied Geography, 26:129-152.

Sáenz, J. y E. Carrillo. 2002. Jaguares depredadores en ganado en Costa Rica: ¿un problema sin solución? Pp. 127-137, en: El jaguar en el nuevo milenio: una evaluación de su condición actual, historia natural y prioridades para su conservación (R.A. Medellín, C. Equihua, C.L.B. Chetkiewics, P.G. Crawshaw Jr., A. Rabinowitz, K.H. Redford, J.G. Robinson, E.W. Sanderson y A. Taber, eds.). Fondo de Cultura/Universidad Nacional Autónoma de México/Wildlife Conservation Society, México, D.F.

Sánchez R.L. y S. Rebollar. 1999. Deforestación en la Península de Yucatán, los retos que enfrentar. Maderas y Bosques, 5:3-17.

Sanderson, E.W., K.H. Redford, C. Chetkiewicz, R.A. Medellín, A.R. Rabinowitz, J.G. Robinson y A.B. Taber. 2002. Planning to save a species: the case for the jaguar, Panthera onca. Conservation Biology, 16:58-72. 
Secaira, F. y O. Maldonado (eds.). 2006. Una agenda para hoy: las acciones que debemos emprender: Plan Ecorregional de las selvas Maya, Zoque y Olmeca. The Nature Conservancy (TNC), San José, Costa Rica.

Secretaría de Medio Ambiente, Recursos Naturales y Pesca (SEMARNAP). 2000. Programa de manejo de la Reserva de la Biosfera de Calakmul. Secretaria del Medio Ambiente, Recursos Naturales y Pesca, Instituto Nacional de Ecología. México D.F.

Secretaría de Medio Ambiente, Recursos Naturales y Pesca (SEMARNAP)-Subsecretaría de Recursos Naturales, Instituto Nacional de Estadística, Geografía e Informática (INEGI)Dirección General de Geografía (eds.) y Universidad Nacional Autónoma de México (UNAM)-Instituto de Geografía (comp.). 2000. Inventario Forestal Nacional 2000-2001. Escala 1:250 000. Secretaría de Medio Ambiente, Recursos Naturales y Pesca (SEMARNAP), Instituto Nacional de Estadística, Geografía e Informática (INEGI), Universidad Nacional Autónoma de México (UNAM), Distrito Federal, México.

Treves, A. y K.U. Karanth. 2003. Human-Carnivore conflict and perspectives on Carnivore management worldwide. Conservation Biology, 17:1491-1499.

Vester, H.F.M., D. Lawrence, J.R. Eastman, B.L. Turner II, S. Calme, R. Dickson, C. Pozo y F. Sangerman. 2007. Land change in the Southern Yucatán and Calakmul Biosphere Reserve: effects on habitat and biodiversity. Ecological Applications, 17:989-1003.

Woodroffe, R. 2000. Predators and people using human densities to interpret declines of large carnivores. Animal Conservation, 3:165-173.

Zarza, H. 2008. Uso de hábitat del jaguar (Panthera onca) en un paisaje influenciado por actividades humanas en el sur de la Península de Yucatán. Tesis de Maestría, Instituto de Ecología, Universidad Nacional Autónoma de México.

Zarza, H., C. Chávez y G. Ceballos. 2007. Uso de hábitat del jaguar a escala regional en un paisaje con actividades humanas en el sur de la Península de Yucatán. Pp. 101-110, en: Conservación y manejo del jaguar en México: estudios de caso y perspectivas (G. Ceballos, C. Chávez, R. List y H. Zarza, eds.). Conabio - Alianza WWF- Telcel - Universidad Nacional Autónoma de México, México.

Zimmermann, A., M.J. Walpole y N. Leader-William. 2005. Cattle rancher's attitudes to conflicts with jaguar Panthera onca in the Pantanal of Brasil. Oryx, 39:406-412. 


\section{APÉNDICE}

Registros de Depredación en la Península de Yucatán, se consideraron todos los registros verificados de ataque, por los autores, por las autoridades del gobierno (e.g. PROFEPA), personal autorizado de las organizaciones no gubernamentales, Biocenosis A.C. y Amavisi A.C., en el estado de Yucatán, y comunicación personal de informantes con experiencia sobre depredación de carnívoros en la región.

No disponible, cercanías de la Reserva Estatal "Bocas de Dzilam" 2125’ N, 88 40’ W; 2. Mano Poderosa, Ejido Presidente Kennedy, Mpio de Panabá, $21^{\circ} 28^{\prime} 56.6^{\prime \prime}$ N, $88^{\circ}$ 29'51.5' ' W; 3. San Antonio, Mpio de Hunucmá, 2106'10.5', N, 9001'13.3'’ W; 4. Rancho La Monedita de Oro, Mpio. de Celestún, 2052'29’’ N, 90¹4'58'’ W; 5. Oxpehool, Km77 Carretera Mérida-Celestún, Mpio. de Hunucmá, 2049’11’' N, 90¹3’58'’ W; 6 y 7. Reserva de la Biosfera Ría Lagartos, sitio de anidación enfrente del Petén Hu de la Reserva de Ría Lagartos, 20²9'55'’ N, 87³8'23', W, 2050’47’' N, 87³8'53' ' W; 8. Chunchucum, 11.9 Km. al Norte del poblado de Hunucmá, Mpio de Hunucmá, 2101’ N, 8951’30’’ W; 9. Celestún, Km 74, Mpio de Celestún, 20¹5’ N, 90²4'54' ' W; 10. Kinchil-Hunucmá, Reserva de la Biosfera Ría Celestún, 2109’ N, 90²4' W; 11. Becanchen, a 75 Km de Tekax, 2003’ N, 89¹3'17' ' W; 12. Rancho Yaxkel, carretera a Uxmal, desviación a Unidad López portillo, a 8 Km de la unidad Xmatuy, Mpio de Muna, 20²7’34’’ N, 8949'25'’ W; 13. Rancho San Isidro, carretera Panabá Yalsihon, Mpio Dzilam de Bravo, 2124'17.2'’ N, 88²7'56.6'” W; 14 y 15. San Lorenzo, Km. 7 carretera Uxmal San Simón, Mpio de Muna, 20¹8'12'’ N, 8948'6' ' W; 20¹9'5.2', N, 8948'11', W; 16. Nuevo Tezoco, Mpio de Tizimin, 21¹4'45.4'’ N, 87³2'15'” W; 17.

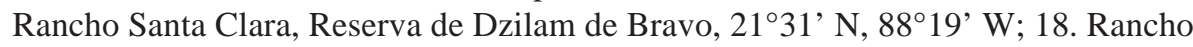
Cuatro Hermanos, Km 67 carretera Mérida - Celestún a 4 km al Sur de la Carretera, 2049'11.3'’ N, 90¹3'59'’ W; 19. Rancho Xcanageler, Oxcutzcab, tomando la carretera a Xul, 2007'32'’ N, 89²9’07'’ W; 20. Hunucmá, aprox. en el Km 10 de la carretera de Hunucma a Sisal, 20¹2'39'’ N, 8957' W; 21. Kinchil, 2052'49'’ N, 9008'58'’ W; 22. Aproximadamente a $4 \mathrm{~km}$ de Chunchucmil, 2040'22' ' N, 90¹3'53', W; 23 . Rancho Cenote Sábalo, Mpio. de Tizimin, 2125'4.3’’ N, 89³5'48.3’’ W; 24. CAMPECHE: Ejido El Refugio, Mpio. de Calakmul, 1848'12', N, 89²2'39' ' W; 25. Al pie de la carretera Dzibalchen-Xpujil, Mpio de Calakmul, 1904'52'’ N, 89¹9’69’’ W. 